\title{
The History and Future of Unlinked Total Elbow Arthroplasty
}

\author{
Takuji Iwamoto, ${ }_{1}^{1}$ Hiroyasu Ikegami, ${ }^{2}$ Taku Suzuki, ${ }^{1}$ Satoshi Oki, ${ }^{1}$ Noboru Matsumura, ${ }^{1}$ Masaya Nakamura, ${ }^{1}$ \\ Morio Matsumoto ${ }^{1}$ and Kazuki Sato ${ }^{1}$ \\ ${ }^{1}$ Department of Orthopaedic Surgery, Keio University School of Medicine, Tokyo, Japan \\ ${ }^{2}$ Department of Orthopaedic Surgery, Toho University, Tokyo, Japan
}

(Received for publication on June 8, 2017)

(Revised for publication on August 7, 2017)

(Accepted for publication on August 10, 2017)

(Published online in advance on August 24, 2017)

\begin{abstract}
Unlinked total elbow arthroplasty (TEA), which has no mechanical connection between the humeral and ulnar components, has theoretical advantages based on its near-normal elbow kinematics and the preservation of bone stock. Unlinked TEA is appropriate only for patients who have limited bone loss or limited deformity and good ligamentous function. This is because postoperative instability has been a major complication of unlinked prostheses. The concept and goal of unlinked TEA is to share the loading stress on the bone implant interface with the surrounding tissues. Although the loosening rate of unlinked prostheses theoretically should be lower than that of linked prostheses (which have a mechanical connection between the humeral and ulnar components), there is no clear evidence that unlinked TEAs are superior to linked TEAs in this respect. However, we believe that primary TEA should be performed using an unlinked TEA, especially for younger patients, because revision surgery for unlinked TEA results in longer prosthesis survival than revision surgery for linked TEA. Improvement of the design of unlinked prostheses and the introduction of less invasive surgical techniques are required to reduce postoperative instability. (DOI: 10.2302/kjm.2017-0007-IR; Keio J Med 67 (2) : 19-25, June 2018)
\end{abstract}

Keywords: total elbow arthroplasty, unlinked type, linked type, postoperative instability

\section{Introduction}

Total elbow arthroplasty (TEA) is indicated for rheumatoid arthritis, osteoarthritis, post-traumatic arthritis, and distal humeral fractures. In the past, TEA prostheses were categorized as constrained, semi-constrained, or unconstrained according to the prosthesis design. Because various linkage mechanisms have recently been developed and because even so-called "surface replacement" prostheses are known to possess some element of intrinsic constraint (as a result of the shape and interaction of their articular surfaces), ${ }^{1}$ the terms "linked type" (with a mechanical connection between the humeral and ulnar components) and "unlinked type" (with no connection between the humeral and ulnar components) are now widely used. ${ }^{2}$ Unlinked TEA has theoretical advantages based on its near-normal elbow kinematics and the pres- ervation of bone stock. However, unlinked TEA is appropriate only for patients who have limited bone loss and good ligamentous function because postoperative instability is a major complication. ${ }^{3}$ The presence of functioning elbow flexor and extensor muscles is also important to maintain the stability of unlinked implants. Elbows with severe joint destruction and unstable elbows should be treated with linked TEA. This article focuses on the history, surgical techniques, surgical results, and problems associated with unlinked TEA.

\section{History}

Aseptic loosening of linked total elbow arthroplasties is a significant problem seen in long-term follow-up, particularly in young and active patients. ${ }^{4}$ The concept and goal of unlinked TEA is to share the loading stress on the 
joint surface/bone implant interface with the surrounding tissues such as the capsule, ligaments, and muscles. Although the loosening rate of unlinked prostheses should theoretically be lower than that of linked prostheses, there has been no definite evidence of this in clinical studies. ${ }^{5-7}$

A number of designs of unlinked TEA prostheses have been developed and employed. The capitellocondylar implant is one of the most studied and widely used unlinked elbow prostheses. ${ }^{8}$ The initial non-stemmed designs were superseded by stemmed prostheses to prevent subsidence of the humeral component. The Souter-Strathclyde total elbow arthroplasty has components that are similar to the anatomy of the ulnotrochlear joint. The prosthesis is unlinked but has a relatively constrained design. ${ }^{9}$ Instability of this implant has been less frequent than that of other unlinked designs; however, loosening of the shortstemmed humeral component has a 3.6- to 5.6-fold risk of revision for aseptic loosening compared to the longstemmed humeral components. ${ }^{10}$ The Kudo total elbow arthroplasty was introduced in $1972 . .^{11,12}$ Initially, both the Type 1 and Type 2 prostheses had non-stemmed humeral components with a cylindrical articulation; however, the humeral component was redesigned to a stemmed component because of a high incidence of early loosening. ${ }^{12}$ The humeral articulation has a saddle design that allows for medial-lateral translation of the ulnar component. The reported results with the current design are favorable, although instability has been problematic because of the low intrinsic stability compared with other designs. ${ }^{13-15}$

The majority of unlinked TEAs lack a radial head prosthesis; indeed, only a few designs have a radial head prosthesis. ${ }^{8}{ }^{816}$ Although, in theory, the radial head component improves soft tissue balance and has been shown to improve stability in in vitro biomechanical studies, ${ }^{17}$ the in vivo results for TEAs using prostheses with radial head components have not been favorable. The radial head prosthesis in the original capitellocondylar total elbow prosthesis was abandoned because it was considered to contribute to the loosening of the humeral component. ${ }^{8}$ The results for the Pritchard ERS prosthesis, which has a radial head component, showed a high failure rate of $70 \%$ after an average of 83 months. Correct positioning and orientation of the radial component was difficult to achieve during surgery. If congruous radiocapitellar articulation was not achieved, severe polyethylene wear of the radial head component or dissociation of the polyethylene was observed. If unlinked TEAs that incorporate radial head components are to achieve future success, precise instrumentation and methodology will be required. ${ }^{18}$

In our opinion, the majority of patients can be managed with an unlinked TEA for their primary arthroplasty; however, some patients do require a linked prosthesis because of a lack of adequate bone stock or functional collateral ligaments. Although a patient's suitability for an unlinked TEA can be predicted preoperatively in most cases, it is always desirable to make a linked implant available in the operating room when a surgeon is planning to perform an unlinked TEA. Postoperative instability is a problem common to all unlinked TEA prostheses, and such instability is often difficult to manage. ${ }^{19,20}$ The K-NOW total elbow system (Teijin Nakashima Medical Co., Ltd., Okayama, Japan), which has been in clinical use since 2005, is a convertible design that provides the option to change an unlinked TEA to a linked type without removing the humeral stem (Fig. 1). Conversion can be performed at the time of the initial arthroplasty or at a subsequent revision procedure (Fig. 2). The removal of a well-fixed humeral stem, either cemented or uncemented, is usually difficult and entails a significant risk of complications. $^{19}$

\section{Surgical Techniques}

The surgical techniques involved in TEA with an unlinked prosthesis depend on the design of the selected prosthesis and the surgeon's preferences. We describe our preferred technique using the K-NOW prosthesis.

The patient is positioned in a lateral position with the affected elbow resting on an arm support. A tourniquet is placed on the upper arm, and a posterior, slightly laterally curved skin incision is made to the elbow. The ulnar nerve is isolated and transposed anteriorly.

The management of the triceps is based on the surgeon's preferences. A distally based triceps flap is commonly used, but recently we have tried to preserve the insertion of the triceps tendon into the olecranon by using the triceps-on approach. ${ }^{21,22}$ Then, the medial collateral ligament is divided and the elbow is dislocated. The radial head is excised, but the lateral collateral ligament is preserved. It is important to preserve the anterior capsule to prevent postoperative dislocation. The resection of the joint surface and the preparation of the stem is performed according to the manufacturer's instruction. The bone resection required to fit the K-NOW prosthesis is minimal, which may facilitate later revision (Fig. 3).

It is important to evaluate the soft tissue balance and articular tracking by suturing the triceps tendon at the trial reduction (Fig. 4). Intraoperatively, if there is some concern about possible instability, conversion to the K-NOW SNAP IN prosthesis (linked type) is easily achieved with additional bone resection.

After placement of the prosthesis and reduction of the elbow, the triceps is meticulously repaired. The medial collateral ligament is repaired if possible without excessive tension. A removable splint is applied at $90^{\circ}$ of flexion for 2 weeks postoperatively. Active and assisted range-of-motion exercises are started 1 week after surgery. Patients are allowed to return to daily living activities 4 weeks after surgery, but are restricted to lifting no more than $2 \mathrm{~kg}$. 


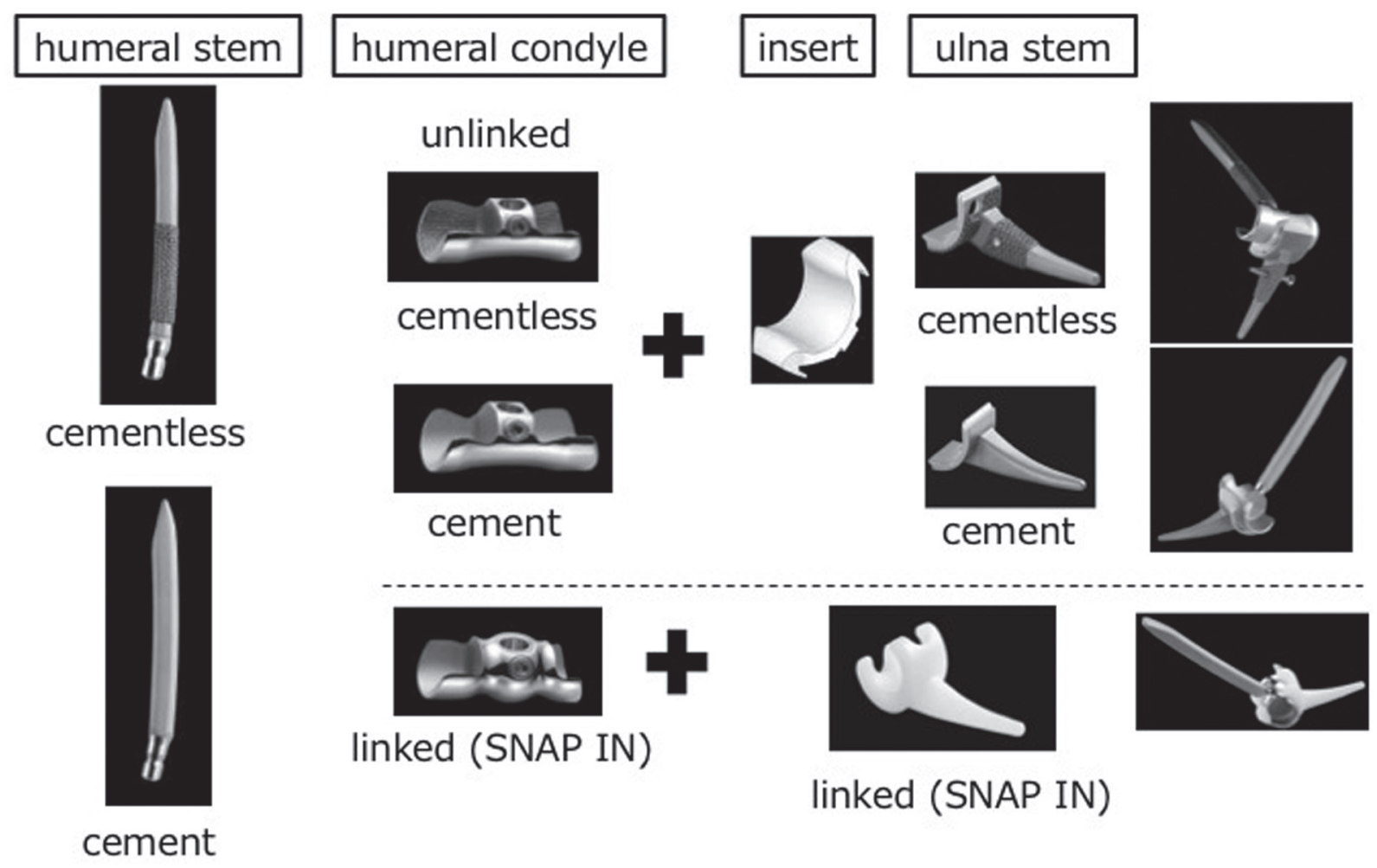

Fig. 1 Component design of the K-NOW total elbow system.

This system uses a modular and convertible design that allows the surgeon to decide during surgery whether or not to use cement and whether to use the unlinked type or linked type. The photos of the prosthesis were provided by Teijin Nakashima Medical Co., Ltd.

\section{Outcomes for Unlinked TEAs}

In 1980, Kudo et al. reported the clinical results for Type 1 and Type 2 Kudo prostheses, which are stemless resurfacing prostheses. ${ }^{11}$ Long-term follow-up revealed that subsidence or loosening of the humeral component was more frequent than had been previously reported. As a result, the Kudo prosthesis was modified to Type 3, which has an intramedullary stem. ${ }^{12}$ In 2001, Tanaka et al. reported successful long-term outcomes, with a $90 \%$ survival rate at 16 years using the Type 3 prosthesis. ${ }^{15}$ Tanaka et al. also mentioned that no great differences in results were found with or without preservation of the anterior oblique component of the ulnar collateral ligament.

The short-term results of the unlinked K-NOW prosthesis in 42 patients with an average follow-up of 24 months were mostly satisfactory. Average elbow flexion improved from $105^{\circ}$ before surgery to $128^{\circ}$ at the final follow-up. Average elbow extension also improved from $-35^{\circ}$ to $-25^{\circ}$; however, postoperative flexion contracture persisted. ${ }^{23}$

There are few studies comparing the outcomes of different unlinked TEA prostheses. Little et al. ${ }^{6}$ compared the outcome of the Souter, Kudo, and Coonrad-Morrey designs in a prospective cohort study based on the year of surgery. At an average follow-up time of 5 years, all three implants relieved pain, and the improvements in the range of motion were comparable among the three groups. The survival rate of the Souter-Strathclyde prosthesis appeared to be worse than those of the other two implants, with 5-year survival rates of $93 \%$ for the Kudo, $90 \%$ for the Coonrad-Morrey, and $85 \%$ for the SouterStrathclyde. Park et al. reported the long-term results of different types of TEA with an average of 13 years of follow-up. They compared complications and revision rates according to the type of implant and found that the complications and revision rates were significantly higher in the unlinked group $(62.9 \%, 34.3 \%)$ than in the linked group $(30.6 \%, 22.4 \%){ }^{24}$

Levy et al. ${ }^{7}$ evaluated the survival of unlinked and linked designs in primary and revision surgeries. The initial survival rate was lower for unlinked prostheses $(56 \%$ at 367 months) than for the linked prosthesis $(84 \%$ at 371 months). However, revision surgery in the linked cohort had a lower survival rate (66\% at 288 months) than that of revision surgery in the unlinked cohort $(72 \%$ at 284 months). Unlinked devices revised to linked devices (84\% at 271 months) were more reliable than unlinked devices revised to another unlinked device (47\% at 284 months). Although the long-term survival of linked TEA prosthe- 

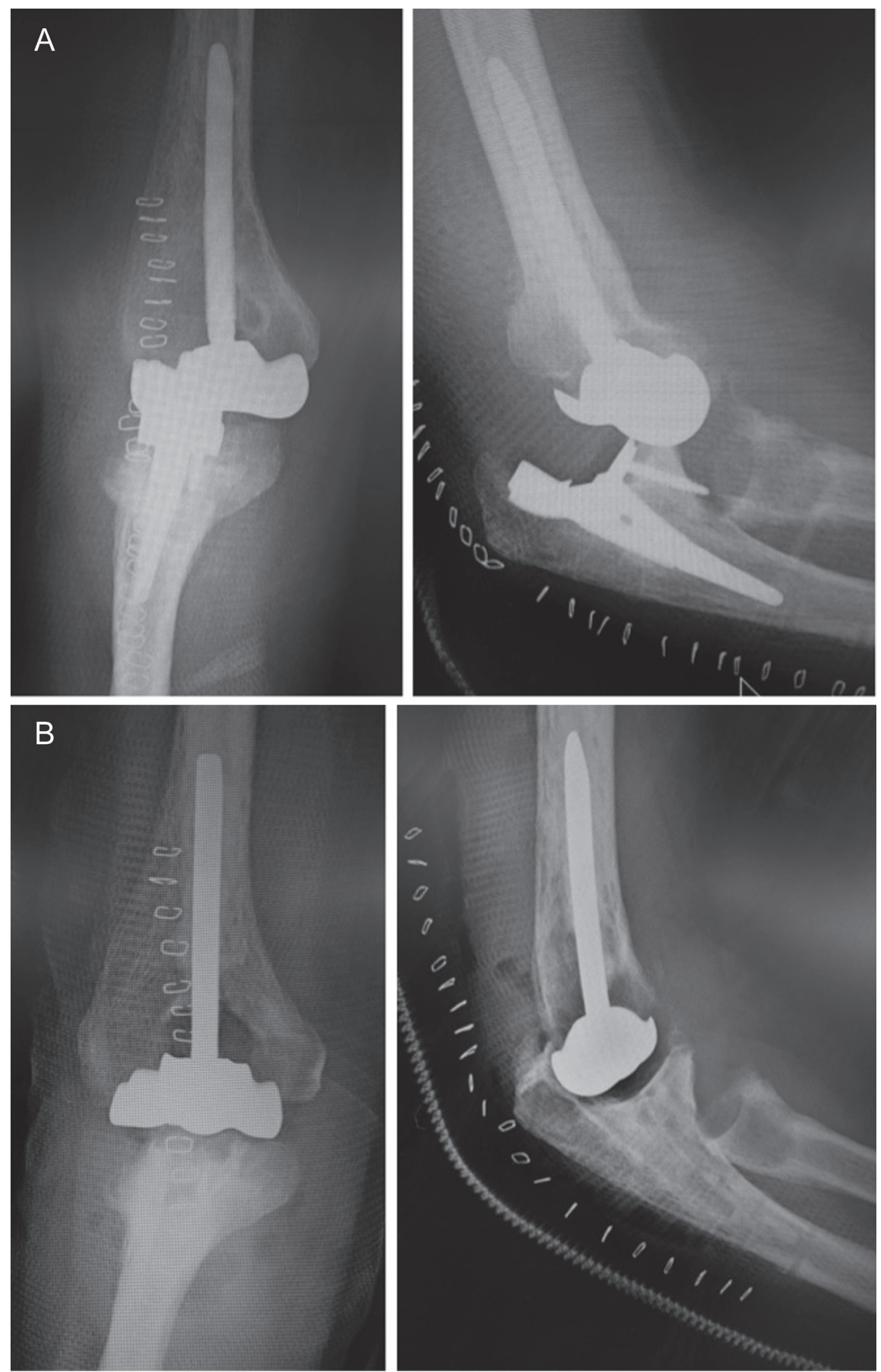

Fig. 2 Conversion from the unlinked type to the linked type is not difficult even as a subsequent revision procedure.

(A) An unlinked K-NOW TEA was dislocated 1 week after surgery. Left, antero-posterior view; right, lateral view. (B) The prostheses were revised to the linked K-NOW SNAP IN TEA without any significant complications such as periprosthetic fracture. Left, anteroposterior view; right, lateral view. 


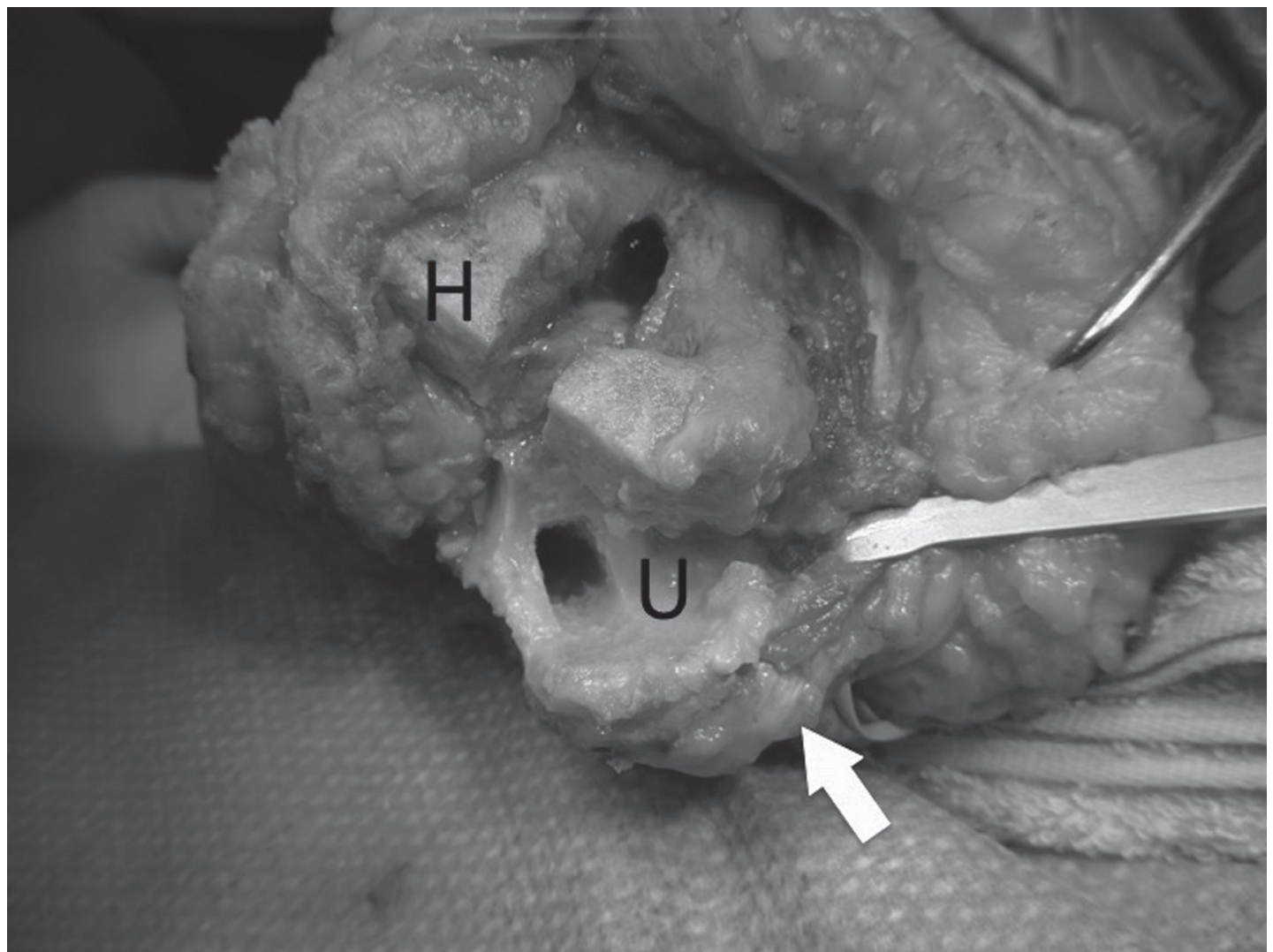

Fig. 3 Photo taken after excision of the joint surface for the K-NOW TEA.

The amount of bone resection is minimal and the triceps tendon (white arrow) was left attached to the olecranon in this patient. H, humerus; U, ulna.

ses is quite acceptable for the primary surgery, unlinked TEA prostheses should be selected especially for younger and more active patients because of the poorer outcome of revision surgery for primary linked TEA prostheses.

\section{Treatment of Postoperative Instability}

Postoperative instability is a major problem common to all unlinked elbow arthroplasties. If instability occurs shortly after surgery, a closed reduction and cast immobilization may maintain reduction. However, conservative treatment often fails and revision surgery is required in most cases. ${ }^{25}$

Ring et al. ${ }^{19}$ reported the operative treatment of unstable capitellocondylar TEAs in 12 patients. Three elbows underwent conversion to linked prostheses. In the other nine elbows, an attempt was made to continue with an unlinked prosthesis: three had reconstruction of one or both collateral ligaments, four had component revision, and two had both ligament reconstruction and component revision. At an average follow-up of 6 years, only three elbows had retained a functioning unlinked prosthesis and four elbows had been converted to a linked prosthe- sis. Thus, 7 of 12 elbows eventually were converted to a linked prosthesis. The authors concluded that the revision of an unlinked total elbow prosthesis to a linked total elbow prosthesis restores elbow function but is technically difficult and there is a high risk of perforation of the humerus and ulna. We always try to stabilize unlinked prosthesis by soft tissue repair before converting to a linked prosthesis. The rotation of the components should be checked carefully because component malposition has been demonstrated to alter the in vitro kinematics and stability of unlinked TEAs. ${ }^{26}$ In our opinion, surgeons who use unlinked TEAs should always take account of the possibility of future revision surgery to a linked prosthesis. We have successfully treated three patients by converting from an unlinked K-NOW prosthesis to a linked K-NOW SNAP IN prosthesis. The conversion was performed during the initial surgery in one case, and the other two were performed at 2 weeks and at 2 months after initial surgery. For this reason, we usually use a convertible design such as the K-NOW TEA.

Previous radial head resection and synovectomy is reported to increase the risk of instability after unlinked TEA. ${ }^{27}$ In the study by Schemitsch et al. that compared 


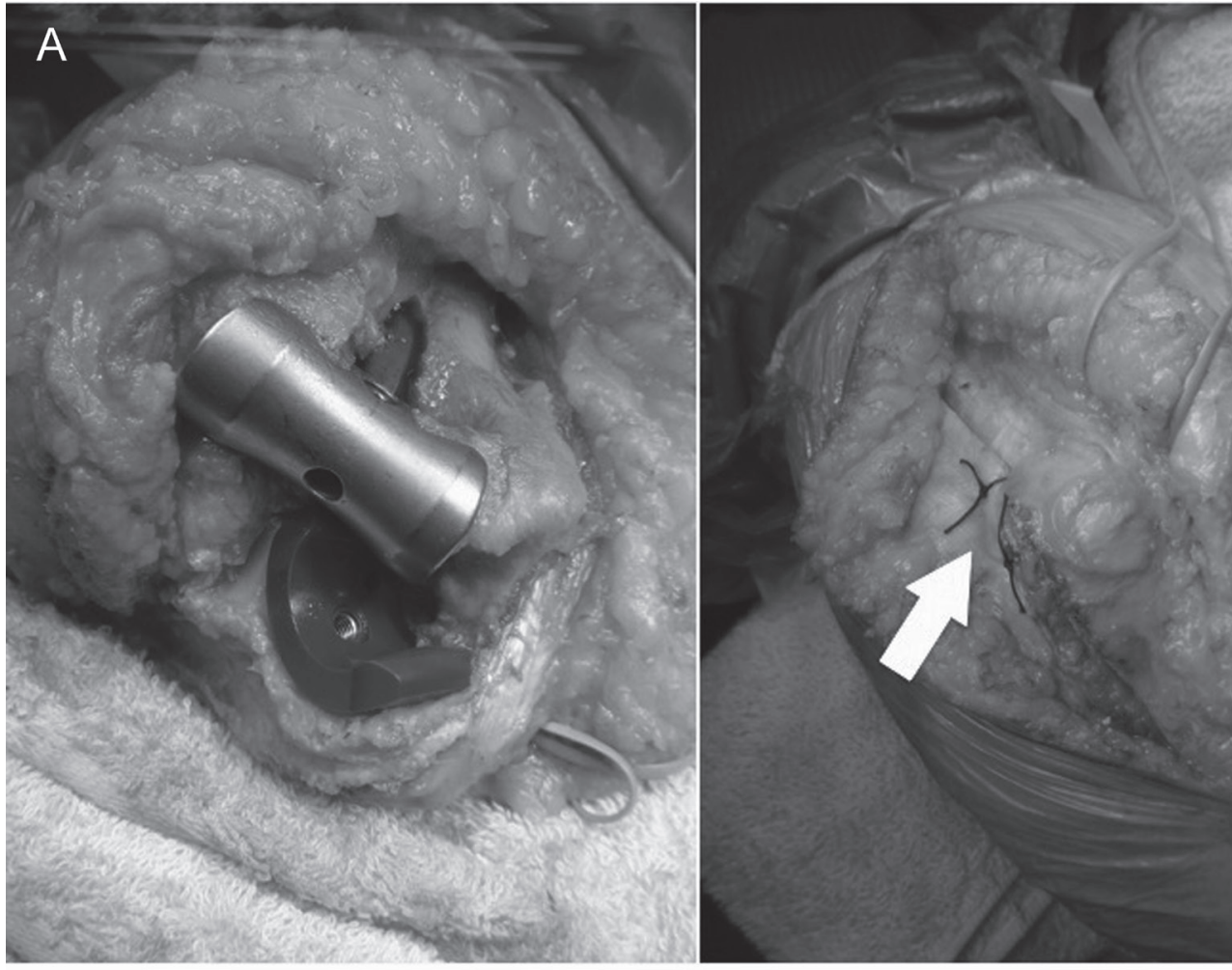

B

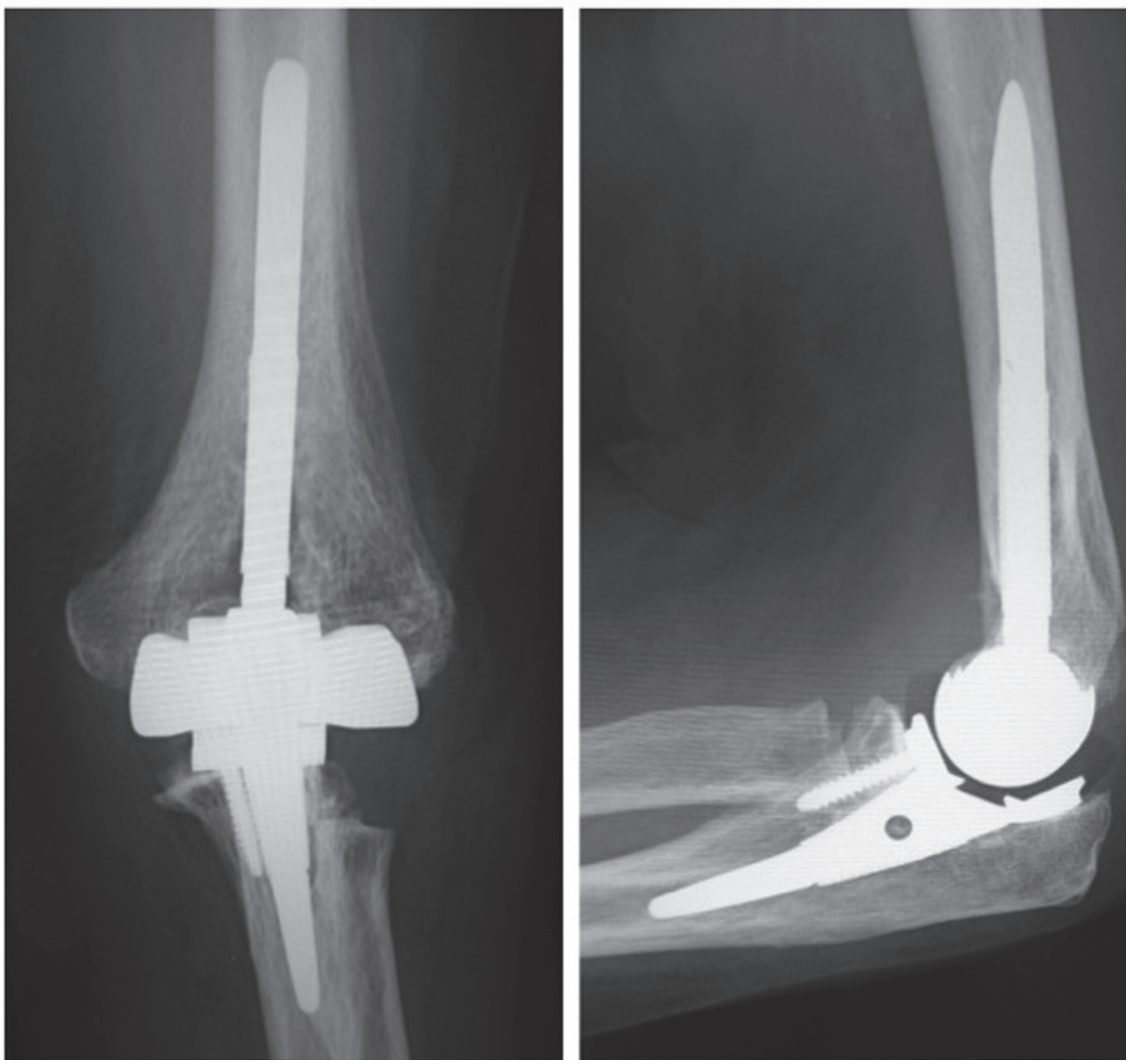

Fig. 4 (A) After trial components are positioned (left), it is important to evaluate the soft tissue balance and articular tracking by suturing the triceps tendon (right, white arrow). (B) Postoperative radiograph. 
primary capitellocondylar TEA with capitellocondylar TEA after failed radial head resection and synovectomy, none of the primary capitellocondylar TEAs dislocated postoperatively, whereas TEAs in 6 of 23 patients with failed synovectomy and radial head resection dislocated postoperatively. The reason for this difference is unclear, but it is important to prepare a linked prosthesis or a convertible design for surgery in these patients.

\section{Summary}

There is no clear evidence that unlinked TEAs are superior to linked TEAs. However, we believe that unlinked TEAs should be considered for primary TEA, especially for younger patients, because of the poor outcome of revision surgery for primary linked TEA. Improvement of the design of unlinked prostheses and the introduction of less invasive surgical techniques are required to reduce postoperative instability. Further clinical trials are also needed to compare the long-term outcomes between unlinked and linked TEAs.

\section{References}

1. Kamineni S, O'Driscoll SW, Urban M, Garg A, Berglund LJ, Morrey $\mathrm{BF}$, An $\mathrm{KN}$ : Intrinsic constraint of unlinked total elbow replacements - the ulnotrochlear joint. J Bone Joint Surg Am 2005; 87: 2019-2027. PMID:16140818, DOI:10.2106/JBJS.C.00983

2. Szekeres M, King GJ: Total elbow arthroplasty. J Hand Ther 2006; 19: 245-253. PMID:16713871, DOI:10.1197/j.jht.2006.02.010

3. Voloshin I, Schippert DW, Kakar S, Kaye EK, Morrey BF: Complications of total elbow replacement: a systematic review. J Shoulder Elbow Surg 2011; 20: 158-168. PMID:21134667, DOI:10.1016/j.jse.2010.08.026

4. Hildebrand KA, Patterson SD, Regan WD, MacDermid JC, King GJ: Functional outcome of semiconstrained total elbow arthroplasty. J Bone Joint Surg Am 2000; 82-A: 1379-1386. PMID:11057465, DOI:10.2106/00004623-200010000-00003

5. Little CP, Graham AJ, Carr AJ: Total elbow arthroplasty: a systematic review of the literature in the English language until the end of 2003. J Bone Joint Surg Br 2005; 87: 437-444. PMID:15795188, DOI:10.1302/0301-620X.87B4.15692

6. Little CP, Graham AJ, Karatzas G, Woods DA, Carr AJ: Outcomes of total elbow arthroplasty for rheumatoid arthritis: comparative study of three implants. J Bone Joint Surg Am 2005; 87: 2439-2448. PMID:16264119

7. Levy JC, Loeb M, Chuinard C, Adams RA, Morrey BF: Effectiveness of revision following linked versus unlinked total elbow arthroplasty. J Shoulder Elbow Surg 2009; 18: 457-462. PMID:19393936, DOI:10.1016/j.jse.2008.11.016

8. Ewald FC, Scheinberg RD, Poss R, Thomas WH, Scott RD, Sledge CB: Capitellocondylar total elbow arthroplasty. J Bone Joint Surg Am 1980; 62: 1259-1263. PMID:7440605, DOI:10.2106/00004623-198062080-00004

9. Khatri M, Stirrat AN: Souter-Strathclyde total elbow arthroplasty in rheumatoid arthritis: medium-term results. J Bone Joint Surg $\mathrm{Br}$ 2005; 87: 950-954. PMID:15972909, DOI:10.1302/0301620X.87B7.15588

10. Ikävalko M, Tiihonen R, Skyttä ET, Belt EA: Long-term survival of the Souter-Strathclyde total elbow replacement in patients with rheumatoid arthritis. J Bone Joint Surg Br 2010; 92: 656-660. PMID:20436002, DOI:10.1302/0301-620X.92B5.22613
11. Kudo H, Iwano K, Watanabe S: Total replacement of the rheumatoid elbow with a hingeless prosthesis. J Bone Joint Surg Am 1980; 62: 277-285. PMID:7358758, DOI:10.2106/00004623198062020-00015

12. Kudo H, Iwano K: Total elbow arthroplasty with a non-constrained surface-replacement prosthesis in patients who have rheumatoid arthritis. A long-term follow-up study. J Bone Joint Surg Am 1990; 72: 355-362. PMID:2312530, DOI:10.2106/00004623199072030-00006

13. Kudo H, Iwano K, Nishino J: Total elbow arthroplasty with use of a nonconstrained humeral component inserted without cement in patients who have rheumatoid arthritis. J Bone Joint Surg Am 1999; 81: 1268-1280. PMID:10505523, DOI:10.2106/00004623199909000-00008

14. Mori T, Kudo H, Iwano K, Juji T: Kudo type-5 total elbow arthroplasty in mutilating rheumatoid arthritis: a 5- to 11-year followup. J Bone Joint Surg Br 2006; 88: 920-924. PMID:16798996, DOI:10.1302/0301-620X.88B7.17356

15. Tanaka N, Kudo H, Iwano K, Sakahashi H, Sato E, Ishii S: Kudo total elbow arthroplasty in patients with rheumatoid arthritis: a long-term follow-up study. J Bone Joint Surg Am 2001; 83-A: 1506-1513. PMID:11679601, DOI:10.2106/00004623-20011000000008

16. Pritchard RW: Anatomic surface elbow arthroplasty. A preliminary report. Clin Orthop Relat Res 1983; 223-230. PMID:6617021

17. Inagaki K, O'Driscoll SW, Neale PG, Uchiyama E, Morrey BF, An KN: Importance of a radial head component in Sorbie unlinked total elbow arthroplasty. Clin Orthop Relat Res 2002; 123 131. PMID:12072754, DOI:10.1097/00003086-200207000-00016

18. van Riet RP, Morrey BF, O'Driscoll SW: The Pritchard ERS total elbow prosthesis: lessons to be learned from failure. J Shoulder Elbow Surg 2009; 18: 791-795. PMID:19278876, DOI:10.1016/j. jse.2008.11.014

19. Ring D, Kocher M, Koris M, Thornhill TS: Revision of unstable capitellocondylar (unlinked) total elbow replacement. J Bone Joint Surg Am 2005; 87: 1075-1079. PMID:15866972, DOI:10.2106/00004623-200505000-00020

20. Chiodo CP, Terry CL, Koris MJ: Reconstruction of the medial collateral ligament with flexor carpi radialis tendon graft for instability after capitellocondylar total elbow arthroplasty. J Shoulder Elbow Surg 1999; 8: 284-286. PMID:10389089, DOI:10.1016/ S1058-2746(99)90145-3

21. Studer A, Athwal GS, Macdermid JC, Faber KJ, King GJ. The lateral para-olecranon approach for total elbow arthroplasty. J Hand Surg Am. 2013; 38(11): 2219-2226 e2213.

22. Dachs RP, Fleming MA, Chivers DA, Carrara HR, Du Plessis JP, Vrettos BC, Roche SJ: Total elbow arthroplasty: outcomes after triceps-detaching and triceps-sparing approaches. J Shoulder Elbow Surg 2015; 24: 339-347. PMID:25591460, DOI:10.1016/j. jse.2014.11.038

23. Ikegami H, Tanji A, Horiuchi Y, Takayama S, Seki A, Nakamura T, Momohara S, Toyama Y: K-NOW total elbow system for the rheumatoid patients. Jpn J Surg 2010; 29: 281-289 [Japanese].

24. Park SE, Kim JY, Cho SW, Rhee SK, Kwon SY: Complications and revision rate compared by type of total elbow arthroplasty. J Shoulder Elbow Surg 2013; 22: 1121-1127. PMID:23664747, DOI:10.1016/j.jse.2013.03.003

25. Ring D: Instability after total elbow arthroplasty. Hand Clin 2008; 24: 105-112. PMID:18299024, DOI:10.1016/j.hcl.2007.11.002

26. Itoi E, King GJ, Neibur GL, Morrey BF, An KN: Malrotation of the humeral component of the capitellocondylar total elbow replacement is not the sole cause of dislocation. J Orthop Res 1994; 12: 665-671. PMID:7931783, DOI:10.1002/jor.1100120509

27. Schemitsch EH, Ewald FC, Thornhill TS: Results of total elbow arthroplasty after excision of the radial head and synovectomy in patients who had rheumatoid arthritis. J Bone Joint Surg Am 1996; 78: 1541-1547. PMID:8876582, DOI:10.2106/00004623199610000-00012 